\title{
Теория предмета Эрнста Малли и интенсиональная логика ${ }^{1}$
}

\author{
И. Б. Микиртумов
}

\begin{abstract}
In the article the object-theoretical logical theory of Ernst Mally is investigated. Considered its non-formal grounds, describes the features of theory, and explores the question of its compliance with the classical propositional logic. For realization of the proposed interpretation of non-trivial implication the intensional logic of object is constructed. The overall conclusion is that the logical theory of the object is Mally submitted the version of the formal semantics.
\end{abstract}

Keywords: object theory, formal semantics, Ernst Mally, intensional logic

Теория предмета, созданная в конце XIX - начале XX в. Алексиусом фон Мейнонгом и его учениками, осталась на периферии современной логики. Причины этого разнообразны, а главная из них состоит в том, что в логике возобладали математические методы и математический стиль исследования. Превращение логики из науки философской в математическую и компьютерную привело к концу XX века к новому межеванию сфер компетенции, в ходе которого логики-философы пытаются заново осознать специфику своего предмета. Поэтому становится актуальным восстановление связи с многовековой философскологической традицией и, в частности, с теми теориями рубежа веков, которые были забыты в силу своей тематической или методической неконъюнктурности ${ }^{2}$.

\footnotetext{
${ }^{1}$ Работа выполнена при поддержке РФФИ, грант № 11-06-00206а.

${ }^{2}$ Интерес к австрийской логике возрастает. Укажу несколько посвященных ей недавних конференций: «Writing the Austrian Traditions» (Торон-
} 
В основании теории предмета Мейнонга лежат теория сознания и концепция интенциональности основоположника австрийской философско-логической школы Франца Брентано. Именно это обстоятельство предопределило относительно слабое влияние на Мейнонга и его учеников немецкой и английской традиций, создало условия для появления оригинальных идей, обсуждавшихся, правда, в основном, в кругу адептов школы.

Настоящая статья посвящена логической системе Эрнста Малли ${ }^{3}$ (1879-1944), которого можно считать наиболее значительным логиком среди учеников Мейнонга. Сначала будут кратко представлены содержательные основания и формальные черты логики Малли, изложенной в его книге «Предметнотеоретические основания логики и логистики» (1912) [13], затем мы ответим на вопрос об адекватности логики Малли классической логике. В последнем разделе статьи будет предложена формализация содержательных принципов системы Малли с помощью инструментария современной интенсиональной ${ }^{4}$ логики.

\section{1 Содержательные основания теории Малли}

В содержательном отношении логическая теория предмета Мейнонга представляет собой последовательную реализацию концепции интенциональности основоположника австрийской школы Франца Брентано. Ее суть можно кратко выразить так: с

то, 2000), «Австрия как культурный центр Европы» (Екатеринбург, 2008 и 2011), с секциями, посвященными философии языка, «Austrian Thought at the Turn of the 20th Century» (Арлигнтон, Техас, 2012).

${ }^{3}$ Сведения о Малли, а также соответствующую библиографию можно найти в Стэнфордской философской энциклопедии:

http://plato.stanford.edu/entries/mally/ (последнее обращение 25.10.2013). В России первым исследователем, обратившимся к наследию Малли, был петербургский ученый Евгений Николаевич Суздалев (см.: [3], [4]). Более новые отечественные работы: [1], [7], [12],

${ }^{4}$ Термины «интенция» и «интенЦиональный» (intentional) принадлежат к философскому словарю схоластики и обозначают, например, содержание понятия в отличие от его объема, а также направленность ума на некоторый предмет. Термин «интенСиональный» (intensional) впервые использовал Бертран Рассел для характеристики случаев, при которых эквивалентность пропозициональных функций не ведет к их тождеству, что имеет место, например, в косвенном контексте: «интенсиональный» есть «неэкстенсиональный». 
каждым психическим явлением (актом) соотнесен содержащийся в нем имманентно предмет, относительно которого это явление (акт) имеет место. Если формируется представление, то есть представляемое, если выносится суждение, то предполагаются его компоненты и их связь. Существование такого интенционального предмета есть, прежде всего, существование в мышлении ${ }^{5}$. В теории предположений (Annahme) Мейнонга выделяются два вида мысленного схватывания - суждение и предположение. Последнее определяется как суждение, которое не сопровождается полаганием содержания суждения истинным [14, c. 368$]^{6}$. Как для суждения, так и для предположения существуют объект схватывания и его объектив ${ }^{7}$. Так, если в обоих случаях подразумевается положение дел, выраженное предложением «7 простое число», то «7» играет роль объекта, а положение дел, согласно которому 7 есть простое число - роль объектива. По сути дела объектив Мейнонга совпадает с фрегевским понятием «мысль», которое сначала неявно вводится в его «Begriffschrift» при разделении утверждения и содержания утверждаемого, а позже получает детальную характеристику в работе 1918 года. Еще одним синонимом для термина обгектив может служить термин пропозииия, последовательное использование которого восходит к Бертрану Расселу, а в интенсиональной логике - к Алонзо Чёрчу.

То, что не является объективом, является предметом и в такой роли выступает в суждении и предположении. Познавательный акт, в котором появляется объектив, - это устанавливающий (тетический) акт. Если объектив $\alpha$ установлен, то тем самым установлены все его следствия, схвачены все его частные случаи (факты) и схвачен всякий предмет (объект или объектив)

\footnotetext{
${ }^{5}$ См.: $[6$, c. $115-116]$

${ }^{6}$ «Annahme» Мейнонга по-английски передают как «assumption», а порусски - как «предположение» или «допущение». Первый термин буквально передает смысл «Annahme» - то, что предполагается для возможности чего-то другого; подразумеваются ментальные акты.

${ }^{7}$ Термин Мейнонга «Objektiv» в прямой передаче по-русски звучит не лучшим образом, и более адекватным было бы перевести его как «подразумеваемое положение дел» или даже «пропозиция», но Мейнонг и Малли обыгрывают сочетание Objekt и Objektiv.
} 
$\beta$, выполняющий $\alpha[13$, с. 2]. Все предметы, выполняющие объектив $\alpha$, подпадают под понятие $A$ и являются предметами вида $A$. Малли называет такие предметы «точками значимости» объектива $\alpha$. Совокупность таких предметов образует область значимости объектива $\alpha$ или просто «область» $\alpha$. В свою очередь $\alpha$ выступает в роли определяющего объектива как для понятия $A$, так и для соответствующего класса предметов (его объема), оказываясь содержанием этого понятия. Такое понимание роли объектива может быть распространено с класса предметов на класс множеств предметов, так что если объектив $\alpha$ характеризует отношение межу двумя предметами, то ему будет соответствовать класс пар предметов. Каждая такая пара есть результат операции соединения двух вещей в одну, в элемент области отношения.

Малли исходит из того, что понятия и суждения всегда соответствуют определенным предметам. Законы мышления не есть ни нормативные предписания, ни законы природы, но объективные условия правильности мышления, имеющие свое основание в природе познаваемых предметов - предметов вообще, а не относящихся к конкретным областям знания. В формулах логики, полагает Малли, знаки надо читать не как знаки понятий или суждений («пропозиций»), но как обозначения предметов этих мыслей. Выраженные в формулах отношения этих предметов есть отношения между предметами любых мыслей вообще, и каждое такое отношение образует ядро соответствующего логического закона. Логика есть, поэтому, всеобщая теория предметов [13, с. 51]. При этом, если определенный объектив - это факт или пропозиция, то неопределенный объектив это пропозициональная функция, так что законы логики следует понимать и как законы предикации. Тем самым предметнотеоретическая теория Малли может быть оценена как формальная семантика.

Логика высказываний характеризуется Малли как теория объективов, выражающая их связи по содержанию. Отношения же вещей составляют предмет алгебры классов, т. е. описываются как отношения «объемов». Малли специально обсуждает вопрос о том, нужна ли логика «содержаний» при наличии логи- 
ки «объемов», т. е. фактически ставит вопрос о семантическом отношении, возникающем между формальной теорией и ее формальной онтологией. Вывод Малли состоит в том, что использование логики содержания, т. е. логики объективов позволяет, схватывая многообразное, мыслить совокупности вещей. Этого нельзя достичь при восприятии, которое дает лишь агрегат. Классы появляются вслед за детерминирующими их объективами $[13$, с. 79$]$ - предметами мышления, схватывание которых этому с необходимостью предшествует [13, с. 81]. Тем самым содержание оказывается первичным, а его интерпретация через отношения объемов классов условных вещей возникает вследствие полагания содержания, а не наоборот.

Отношение между содержательной теорией, относящейся к сфере познания - теорией схватывания, - и сферой вещей позволяет прояснить отношение между объективом и вещью. Объективы как содержания сознания суть особого рода предметы, которые выполняются или «фактизируются» в случаях и в вещах или через вещи [13, с. 57]. При непосредственном восприятии вещи или при ее репродукции комплекс объективов присутствует имплицитно, иначе восприятие не было бы возможно. Таким образом, вещь может быть понята как имплицитный детерминат всех своих объективов или, в более современной терминологии, как куст свойств.

Всякий частный случай имплицитно содержит комплекс более простых фактов, причем, если он имеет место в действительности, то это есть «фактизация» совершенного комплекса объективов. Условием такой фактизации оказываются вещи, их свойства и отношения. Вещь в абсолютном смысле - предмет, который имплицитно присутствует во всяком объективе или его отрицании. Так, например, может быть определено тело. Вещью в относительном смысле, т. е. субъектом предикации может быть любой предмет [13, с. 57-58]. Малли подчеркивает, что предикация вещи в абсолютном смысле или предикация телесному первична по отношению к любой другой, так что второпорядковая предикация сводима к первопорядковой ${ }^{8}$. Любой частный слу-

\footnotetext{
${ }^{8}$ Малли не использует этих терминов и не привлекает теорию квантификации.
} 
чай, будучи следствием некоторой причины, сам эквивалентен этой причине - сохраняет ее в себе, существуя как объектив. Поэтому и вещь полностью определяется частным случаем своего существования. Объектив, содержащийся в данном частном случае, называется «выполняющимся в данном случае», можно также сказать, что это есть частный случай объектива. Пример такого случая можно найти в (совершенном) так-бытии, т.е. в том, что свойственно конкретной вещи. Лист бумаги дает случай белизны, протяженности, весомости, отличия от другого листа бумаги, движение брошенного камня есть случай движения. Идет дождь - это случай «дождения» и т. п. [13, с. 57].

Очень любопытна и точна вполне адекватная интенсиональной логике характеристика вынесения и оценивания суждений. Истинность и ложность у Малли - это характеристики объектива, сопоставляемого суждению вида «имеет место, что этот шар бел», а именно мы должны понять, факт перед нами или нет. Вынесению решения об истинности или ложности предшествует схватывание в виде предположения объектива «этот шар бел», после которого схваченное положение дел соотносится с действительностью. Результатом становится новое предположение «имеет место, что этот шар бел», проецируемое теперь на воображаемого субъекта. Лишь в этот момент приходит оценка истинности как адекватности акта утверждения действительности $[13$, с. $61-62]$.

Наиболее важное отношение между объективами - это отношение обусловливания или причинения (в изложении системы Малли мы обозначим его символом $\rightarrow$, а в системе интенсиональной логики - как $\Rightarrow)$. Переход от $\alpha$ как причины к $\beta$ как следствию схватывается как отношение благодаря пониманию содержания объективов, а не через количественные отношения подпадающих под объективы классов вещей: «эта фигура квадрат, значит, она прямоугольник». Такую связь надо отличать от условного суждения «если эта фигура квадрат, то она прямоугольник», которое выносится на основе отношений вещей. Отношению следования соответствует акт, в котором объектив $\beta$ с очевидностью устанавливается на основе интеллектуальной 
диспозиции, возникшей вследствие установления $\alpha$, усматривается «в связи с $\alpha »[13$, с. 66].

Свою основную аксиому $\alpha \rightarrow \alpha$ Малли считает определенным объективом об объективах, т. е. свойством второго порядка. Эта формула не есть поэтому следствие произвольного объектива о вещах, т. е. неверно, что $\beta \rightarrow(\alpha \rightarrow \alpha)$, хотя доказательство $\alpha \rightarrow \alpha$ дается с использованием понятия «объем объектива». Здесь проявляется намерение Малли развести материальную импликацию и отношение обуславливания. Содержание всегда истинного объектива у Малли пусто: «быть или не быть красным» - пограничный случай так-бытия вещи, равноценный отсутствию определенности. Поэтому и объем такого объектива универсален. Все логические законы выражают, поэтому, свойства второго порядка, т. е. свойства объективов, а не вещей [13, с. 69], что делает неверным утверждение обусловливания логическим законом произвольного нелогического по своей природе объектива. Эта несомненно плодотворная идея Малли вступает в противоречие с последовательно отыскиваемой им редукцией второпорядкового к первопорядковому.

Для Мейнонга и Малли важна дифференциация актов схватывания объектива, его предположения и утверждения, поскольку она позволяет различать предметы и вещи, которые могут быть мыслимы, но невозможны, а также мыслимы, возможны, но недействительны. Этим актам соответствуют эпистемические установки и ментальные действия. Так, акту допущения или предположения случая неопределенного (но непротиворечивого) объектива может соответствовать некоторая ясность или очевидность. Очевидности суждения соответствует очевидная фактичность, очевидному предположению - наличие схваченного объектива. При обнаружении треугольника очевидному акту суждения, предшествующему этому обнаружению, соответствует фактичность того, что предмет выполняет свойство треугольности в этом конкретном случае. Если же треугольник предположен (без внутреннего противоречия), то ориентируются на выполнение этого содержания и схватывают просто наличествующий («всеобщий») случай бытия треугольником. В определении понятия «круг» подразумевается индивид, вещь, 
выполняющая содержащиеся в определении объективы. Но то, что непосредственно в этом понятии мыслится, есть предмет понятийный абстракт, который не подразумевает ничего данного, но мыслится чисто. Это не целевой предмет понятия, т. е. не круг и не то, что может попасть в объем понятия «круг», это специфический понятийный предмет [13, с. 63].

Для каждого предмета суждения можно указать сообразное этому суждению очевидное предобнаружение определенности вещи, которому соответствует фактическое выполнение объектива подразумеваемой «вещью», и точно так же очевидному предположению неопределенного объектива некоторой «вещи» соответствует такое же, но простое, т. е. не-фактическое выполнение объектива. Чистому содержанию мысли «нечто, являющееся $A$ »ли «просто мышлению» этой мысли (без акта полагания) соответствует чистая детерминация формы абстрактного «нечто» через детерминатор «быть $A$ ». Полагание идет через абстрактный понятийный предмет к целевому предмету понятия и через детерминат формы к имплицитному детерминату предположенного объектива, к тому, что выполняет или могло бы выполнить объектив [13, с. 64].

Малли поясняет, что в предположении «а делимо на 9, значит, и на 3» такой акт перехода происходит с предположения об $\alpha$ к очевидному предположению, что $\beta$. Здесь отношение следования имеет место не в виде факта, а содержательно. Здесь нет суждения, так как нет предметов суждения, посылки и вывод соединены «в фантазии». Вместе с тем в гипотетическом суждении «если а делимо на 9, то и на 3» имеем подлинное суждение [13, с. 65], поскольку речь идет о случаях делимости на 9, которые оказываются случаями делимости на 3 , что относится к фактам. Здесь причина взята как предположение, но ее связь со следствием составляет определенный объектив. Отличие от «во всех случаях, когда а делится на 9, оно делится и на 3» в том, что эти случаи берутся не как «веи», могущие и не быть, но именно как частные случаи, т. е. как то, что есть.

Формальный предмет - это то, что существует как предмет номинального определения. Его действительное или даже возможное существование не обязательны. Наименьшее по содер- 
жанию существование - в статусе предмета - дается в акте установления содержания понятия. Не всегда этому сопутствует с очевидностью данное предположение (Annahme), как, например, его нет у «круглого квадрата». Законы логики содержатся в каждом частном случае и выполняются каждым существующим предметом, и предметная область логики охватывает все фактически и возможно существующие предметы, а также противоречивые по содержанию, которые наравне с иными предметами имплицитно полагаются при формулировании комплекса объективов - понятия [13, с. 72-73]. Всякий предмет выполняет комплекс объективов, который Малли называет совершенным, и есть при этом фактический определитель этого «совершенства». Но есть предметы, которые являются чистыми детерминатами формы определяемого объектива, но при этом относительно них бессмысленно говорить, что они выполняют или не выполняют произвольный объектив, что должно следовать из закона исключенного третьего. Например, относительно чистого детермината объектива «быть треугольным», т. е. относительно треугольника вообще бессмысленно говорить о том, что он красен или не красен. Такой предмет своим формальным определением дан, поэтому, несовершенно, но относительно объективов, которые он выполняет - совершенно. К числу таких объективов принадлежат, например, объективы «быть формальным детерминатом определения», «быть геометрической фигурой» и пр. Несовершенный предмет подчиняется закону исключенного третьего и закону противоречия. Это ключевая для Малли идея позволяет решить проблему противоречия там, где необходимо мыслить противоречивое. «Быть круглым квадратом» - объектив, т. е. предмет, на предикацию которому тех или иных свойств распространяются законы логики, поэтому как объектив он дан совершенно и существует. Но вещь, являющаяся круглым квадратом, определена этим объективом несовершенно, как онтологическая условность или чистый детерминат, и, в силу противоречивости детерминирующих ее объективов, не существует. Абстракт «то, что есть и не есть $\alpha »$ не конституирует невозможную вещь, невозможны лишь вещи, которые такой объектив выполняют. Сам же абстракт есть существующий предмет. В 
конкретных случаях познания мы устремляемся к фактическим определенностям, к объективам, которые выполняют предметы, являющиеся фактически совершенно определенными вещами, а несовершенные вещи оказываются в этом случае невозможными предметами. Установить, что некоторый объектив противоречив, становится возможным при предположении о его утверждении [13, s. 76-77].

Для Мейнонга [15, s. 176-181] и Малли здесь шла речь о различении конститутивных и внеконститутивных свойств. Требование, согласно которому всякий предмет либо выполняет, либо не выполняет всякое свойство, наталкивается на неопределенность некоторых предметов относительно некоторых свойств, что делает такое требование неконститутивным, т. е. не имеющим отношения к предмету. Так, противоречие возникает при предположении о действительном существовании вещи, которая выполняет противоречивый дефинитивный комплекс, т. е. при его трактовке de re, в конститутивном смысле, когда вещь должна либо выполнять, либо не выполнять всякий объектив. Но при употреблении этого комплекса de dicto, т. е. при его упоминании или цитировании не до конца ясное предположение целевого предмета и схватывание его невозможности само не является ни противоречивым, ни невозможным. Свойства и отношения вещей выразимы объективами, вещь - это то, что может быть определено совершенно, что порождает определенные объективы. Но свойства и отношения объективов специфичны.

Можно видеть, что для реализации этой теории Мейнонга и Малли не будет достаточно предикации второго порядка или же теоретико-типовой дифференциации предикатов по вещам и по объективам. Возможность поместить объектив в косвенный контекст, например, модальный или эпистемический, - это непременное условие его успешной репрезентации в формальной системе.

\section{2 Между классическим и неклассическим следованием}

Малли строил свою логическую систему как универсальную. Перечислим ее компоненты: (1) классическая логика высказыва- 
ний (логика объективов), (2) теория классов, (3) теория понятий, (4) теория равенства, (5) теория подобия, (6) теория приблизительного понимания объектива, (7) теория следования, (8) теория существования и модальности, (9) теория числа, (10) теория измерений, (11) теория вещей. Представить все эти компоненты здесь невозможно за недостатком места, кроме того, нас интересует логика объективов и отношение обусловливания, поэтому ниже будут представлены именно эти фрагменты системы Малли.

Логика на предметно-теоретическом основании строится Малли как теория, включающая уровень языка-объекта и уровень метаязыка - последний соответствует алгебре классов. Постулируются аксиомы, из которых с помощью неформализованных правил выводятся теоремы. При этом обоснования, которые с современной точки зрения следует охарактеризовать как семантические, используются наравне с элементами теории вывода. Так, например, логические связки, которые, по замыслу Малли, должны быть адекватны классической логике, не рассматриваются как истинностные функции. Их свойства представлены теоремами, которые обосновываются семантически, т. е. как выражающие те или иные отношения классов. Для Малли в получении такого обоснования логики высказываний состояла центральная задача, поскольку теория классов воспринимается им как теория вещей - предметов.

Приводимые Малли доказательства теорем опускаем, его не очень удобную нотацию модернизируем и, следуя за текстом книги Малли, сохраняем нумерацию аксиом и теорем.

Алфавит: $a, b, c, d, a_{1}$ - переменные для объективов (фактов, положений дел, пропозиций, все утверждения одного и того же объектива эквивалентны, т. е. за объективом стоит класс эквивалентности предложений естественного языка), $i, j, \ldots$ - переменные по индивидам (вещам), $x, y, z, x_{1}, \ldots$ - переменные для классов вещей, $U$ - универсальный класс, $\varnothing-$ пустой класс, $P$ - класс всех объективов, $\neg, \wedge, \vee, \rightarrow-$ символы, соответственно отрицания, логической суммы, логического произведения, «обусловливания», $\sim, \cap, \cup, \subseteq, \in$ - дополнение, пересечение, объ- 
единение, включение, принадлежность. Скобки используются обычным образом.

Пусть $X, Y, Z, X_{1}, \ldots$ - метапеременные по классам, $\alpha, \beta, \gamma, \delta, \alpha_{1}, \ldots$ - метапеременные по объективам, $I, J, I_{1}, \ldots$ метапеременные по индивидам. Тогда варианты правильно построенных выражений, обозначающих классы, заданы перечнем: $\left|x, y, z, x_{1}, \ldots\right| U|\varnothing| X|\sim X| X \cap Y \mid X \cup Y$, варианты правильно построенных выражений, обозначающих объективы, заданы следующим перечнем: $\left|a, b, c, d, a_{1}, \ldots\right| \alpha|\neg \alpha| \alpha \rightarrow \beta \mid$ $\alpha \wedge \beta|\alpha \vee \beta| A \subseteq B|I \in A| \beta \Vdash \alpha \mid I \Vdash \alpha$. $\alpha$ читается как «объектив $\alpha$ имеет место». $\neg \alpha$ читается как «имеет место не- $\alpha$ » или «имеет место объектив, состоящий в отрицании $\alpha » . \alpha \rightarrow \beta$ читается как « $\alpha$ обусловливает $\beta »$, или как « $\alpha-$ причина $\beta$. $X \subseteq Y$ читается как «множество $X$ включено во множество $Y$ », или « $X$ есть вид $Y »$ или «понятие $X$ подпадает под понятие $Y$ » $\beta \Vdash \alpha$ - читается как «объектив $\beta$ выполняет объектив $\alpha »$. $I \Vdash \alpha-$ читается как «случай $\alpha$ для $I »$ или «индивид $I$ выполняет объектив $\alpha »$.

Ряд понятий вводится определениями:

$(\alpha \leftrightarrow \beta)={ }_{D f}(\alpha \rightarrow \beta) \wedge(\beta \rightarrow \alpha)$ - эквивалентность объектиBов.

$(X=Y)=_{D f}(X \subseteq Y) \wedge(Y \subseteq X)$ - равнообъемность классов. $X \Vdash \alpha$ iff $f_{D f}$ если $(I \in X)$, то $I \Vdash \alpha-\ll$ класс $X$ выполняет объектив $\alpha \gg$.

$\mathrm{T}={ }_{D f} \beta: \alpha \rightarrow \beta$ для любого $\alpha$. Всегда истинный объектив ${ }^{9}$. $F={ }_{D f} \beta: \beta \rightarrow \alpha$ для любого $\alpha$. Всегда ложный объектив ${ }^{10}$.

\footnotetext{
${ }^{9}$ Объектив $T$ выполняется при любых условиях, что, например, верно для законов логики или необходимых равенств арифметики. Малли поясняет, что в случае, когда мы сталкиваемся с $T$, его следование из произвольного $\alpha$ связано не с их собственной связью, а с определением понятия следования. Малли называет объективы такого типа «Нуль-объективами» и обозначает их как «о̃». Следуя более привычным обозначениям, и постольку, поскольку это не сказывается на представлении иных аспектов содержания работы Малли, мы используем знак «T».

${ }^{10}$ Если объектив $F$ выполняется, то выполняется и всякий другой. Это будет верно, например, для отрицаний законов логики, т. е. для противоречий. Малли называет такой объектив противоречивым. Мы обозначаем его привычным образом как «F», то, чего не может быть, «не-факт». См.: $[13$, c. 56].
} 
$X^{\beta}={ }_{D f}\{i: i \in A \wedge i \Vdash \beta\}-$ детерминация $X$ объективом $\beta$.

Если $\alpha \rightarrow \beta$, то $\beta$ зависим от $\alpha$.

Если для $\alpha$ и $\beta$ верно, что $\neg(\alpha \rightarrow \beta)$ и $\neg(\beta \rightarrow \alpha)$, то $\alpha$ и $\beta$ независимы друг от друга.

Если $\neg(\alpha \rightarrow \neg \beta)$, то $\alpha$ и $\beta$ совместимы друг с другом; в противоположном случае - несовместимы.

Определенным называется объектив $\alpha$, для которого имеет место, что либо $\alpha \leftrightarrow T$, либо $\alpha \leftrightarrow F$.

$[\alpha]={ }_{D f}\{\beta: \alpha \rightarrow \beta\}-$ совокупность следствий объектива $\alpha$.

$P^{-[\alpha]}-$ совокупность «неследствий» $\alpha$.

В системе Малли воспроизводятся тавтологии логики высказываний (исключения обсуждаются ниже), законы теории классов, а также свойства равенства. Приведем некоторые специфические аксиомы и теоремы, характеризующие логику Малли как логику отношений между высказываниями об объективах и высказываниями о классах.

Принцип $E$ : если $(\alpha \rightarrow \beta)$, то $(\alpha(i) \rightarrow \beta(i))$. Если полагается объектив $\alpha$, то тем самым (1) имплицитно полагаются все следствия $\alpha,(2)$ схватывается всякий частный случай $\alpha,(3)$ всякий предмет, выполняющий $\alpha$, схватывается как предмет определенного вида.

2. $\alpha \rightarrow \alpha$ (аксиома включения).

8. $(\alpha \rightarrow \beta) \leftrightarrow\left(U^{\alpha} \subseteq U^{\beta}\right)$ (аксиома реципрокности). Это центральная аксиома системы, обеспечивающая связь уровней логики содержания (логика объективов) и логики объемов (алгебра классов).

8.1. $(\alpha \leftrightarrow \beta) \leftrightarrow\left(U^{\alpha}=U^{\beta}\right)$.

18. $U^{\alpha} \cup U^{\beta} \Vdash \alpha \vee \beta$.

20. $U^{\alpha} \cap U^{\beta} \Vdash \alpha \wedge \beta$.

31. $\neg(T \rightarrow F)$.

31.1. $\neg(U \subseteq \varnothing)$.

Таким образом, можно построить ряд последовательно обусловливающих друг друга объективов: $F \rightarrow \ldots \rightarrow \alpha \rightarrow \beta \rightarrow$ $\gamma \rightarrow \ldots \rightarrow T$, и ряд содержащих друг друга классов $\varnothing \subseteq \ldots \subseteq$ $A \subseteq B \subseteq D \subseteq \ldots \subseteq U$.

32.1. $\alpha \wedge \neg \alpha \leftrightarrow F$. 
При ознакомлении с работой Малли поначалу создается впечатление эквивалентности логики объективов классической логике высказываний. Но свойства отношения выполнимости $\Vdash$ и аксиома реципрокности, лежащая в основе многих теорем, делают систему Малли не только логикой объективов, но и логикой отношения выполнимости, так что ее наиболее интересные теоремы выражают зависимость свойств и отношений объективов от свойств и отношений соответствующих им классов. Учитывая, что Малли понимает отношение обусловливания между объективами как причинно-следственную связь не только логической природы, столкновение логики объективов со свойствами материальной импликации становится неизбежным. Малли не рассматривает эти свойства специально, но принимает ряд ограничений, которые призваны сделать отношение обусловливания «релевантным». В чем состоит его цель и какие средства для ее достижения он использует?

Рассмотрим следующие определения Малли.

64.1. Определение родственности по следствиям - подобия: Если $[\gamma] \subseteq[\alpha],[\gamma] \subseteq[\beta],[T] \subset[\gamma]$, или же, если $\Gamma \subseteq P^{-[\alpha]}$, $\Gamma \subseteq P^{-[\beta]},[T] \subset \Gamma$, или имеют место оба соотношения, то объективы $\alpha$ и $\beta$ называются родственными по следствиям или подобными, причем если имеет место только второе соотношение, то они родственны в более широком смысле (по не-следствиям), а если имеет место первое соотношение или оба соотношения, то в более узком смысле. Ограничение $[T] \subset[\gamma]$ принимается Малли с тем, чтобы сделать $\gamma$ нетривиальным объективом. Поскольку для любого $\delta$ верно, что $[T] \subset[\delta]$, родственность $\alpha$ и $\beta$ на основе пересечения областей их следствий по области $[T]$ универсальна, в таком случае все объективы родственны друг другу. Ограничение позволяет указывать родственность только при совпадении нетривиальных следствий.

Если, кроме того, для любого $[\delta]$, такого, что $[\delta] \subseteq[\alpha]$ и $[\delta] \subseteq[\beta]$, верно, что $[\delta] \subseteq[\gamma]$, и при этом для любого $\Delta$, такого, что $\Delta \subseteq P^{-[\alpha]}$ и $\Delta \subseteq P^{-[\beta]}$ верно, что $\Delta \subseteq \Gamma$, то $\alpha$ и $\beta$ называются родственными по следствиям или подобными по набору следствий $[\gamma]$, соответственно, благодаря общему классу 
не-следствий $Г$, соответственно, благодаря обоим. Можно видеть, что $[\gamma]=[\alpha] \cap[\beta]$, а $\Gamma=P^{-[\alpha]} \cap P^{-[\beta]}$.

64.2. Совокупность $[\gamma] \cup P^{-[\alpha]} \cap P^{-[\beta]}$ называется совместными следствиями $\alpha$ и $\beta$ : $G c(\alpha, \beta)=([\alpha] \cap[\beta]) \cup\left(P^{-[\alpha]} \cap P^{-[\beta]}\right)$.

Понятие совместного следствия вводится Малли как инструмент оценки релевантности следования. Если совокупные следствия двух объективов составляют $P$, то такие объективы идентичны, если же эта совокупность равна у них $\varnothing$, то они абсолютно различны. При этом $G c(\alpha, \beta)$ характеризует условия, при которых оказывается выполненным объектив вида $\alpha \rightarrow \beta$. Условие это задается совокупностями объективов, а не предметов, т. е., будучи условием логического следования, не редуцирует условия истинности к условиям семантическим.

64.3. Определения различия по следствиям. Если $\Gamma \subseteq[\alpha]$, $\Gamma \subseteq P^{-[\beta]}$ или $\Lambda \subseteq P^{-[\alpha]}, \Lambda \subseteq[\beta]$, или верно и первое, и второе, то $\alpha$ и $\beta$ называются различными по следствиям или просто различными. Если, кроме того, для любого $\Delta$ такого, что $\Delta \subseteq[\alpha]$ и $\Delta \subseteq P^{-[\beta]}$, верно, что $\Delta \subseteq \Gamma$, то если для любого $\Sigma$ такого, что $\Sigma \subseteq P^{-[\alpha]}$ и $\Sigma \subseteq[\beta]$, верно $\Sigma \subseteq \Lambda$, то $\alpha$ и $\beta$ называются различными по следствиям по $\Gamma$, соответственно, по $\Lambda$, соответственно, по $\Gamma u \Lambda$, т. е. по сумме $\Gamma \cup \Lambda$. Можно видеть, что в этом случае $\Gamma=[\alpha] \cap P^{-[\beta]}$, а $\Lambda=P^{-[\alpha]} \cap[\beta]$.

64.4. Совокупность различающихся следствий $\alpha$ и $\beta$ обозначим как $U c(\alpha, \beta): U c(\alpha, \beta)=\left([\alpha] \cap P^{-[\beta]}\right) \cup\left(P^{-[\alpha]} \cap[\beta]\right)$.

66. $G c(\alpha, \beta)=P-U c(\alpha, \beta)$.

Если $[\alpha] \subseteq[\beta]$ и $[\beta] \subseteq[\alpha]$, то $\alpha$ и $\beta$ назыются «имеющими равные следствия» или просто равными: $\alpha=\beta$. Вопрос о тождестве пропозиций, выраженных утверждениями $\alpha=\beta$ и $\alpha \leftrightarrow \beta$, требует отдельного рассмотрения. Если оценивать объективы по совокупностям их следствий, то очевидно, что из $\alpha=\beta$ будет следовать $\alpha \leftrightarrow \beta$. Кроме того, верна теорема

67. $([\alpha]=[\beta]) \leftrightarrow(\alpha \leftrightarrow \beta)$.

Но нельзя еще утверждать, что из $\alpha \leftrightarrow \beta$ следует $\alpha=\beta$. Могут ли быть нетождественными объективы, имеющие одни и те же следствия и обусловливающие друг друга? Этот вопрос относится уже к сфере интенсиональной логики и Малли отвечает на него, опираясь на содержательную посылку, согласно кото- 
рой тавтологии логики, будучи эквивалентными, не выражают один и тот же объектив.

Доказываются теоремы

67.1. $(\alpha \leftrightarrow \beta) \rightarrow G c(\alpha, \beta)=G c(\alpha, \alpha)=[\alpha] \cup P^{-[\alpha]}=P$.

67.2. $(\alpha \leftrightarrow \beta) \rightarrow U c(\alpha, \beta)=U c(\alpha, \alpha)=\left([\alpha] \cap P^{-[\alpha]}\right) \cup([\alpha] \cap$ $\left.P^{-[\alpha]}\right)=[T]$,

а из теорем 66 и 67.1 следует, что $[T]=\varnothing$. При каких условиях из всегда верного объектива не следует ничего?

Для установления адекватности системы Малли классической логике, коль скоро речь идет о классической теории классов как о семантическом основании, следует выяснить, действительно ли отношение «обусловливать» или «причинять», интерпретирующее $\rightarrow$, соответствует отношению логического следования в классической логике высказываний. Это тем более важно, что Малли определяет совокупность следствий объектива $\alpha$ как множество таких объективов $\beta$, для которых верно $\alpha \rightarrow \beta$, т. е. характеризует совокупность следствий в терминах теории вывода.

Здесь мы сталкиваемся с трудностями не формального, а содержательного характера, а именно с тем, что Малли определяет $G c(\alpha, \beta)$ с помощью понятия родственности по следствиям, в котором, как мы видели выше, $[T]$ исключается из совокупности следствий любого объектива ради достижения большей релевантности. Вследствие этого при определении $G c(\alpha, \beta)$ принимается, что $[T]=\varnothing[13$, с. 26 , определение 68]. Обосновывая это равенство, Малли дает ряд содержательных пояснений. Всегда истинный объектив, с его точки зрения, есть пограничный случай детерминации так-бытия предмета, который не несет в себе полезного содержания и оказывается поэтому «пустым». Это касается законов логики, т. е. суждений общего характера. Для конкретных истинных определенных объективов, например $2+3=5$, Малли использует термин «случай», подразумевая частный случай, при котором неопределенный объектив структуры $\mathrm{x}+y=z$ оказывается истинным. В случае объектива «быть красным или не быть красным» мы имеем определенный объектив и факт, хотя объектив «быть красным» сам по себе не является определенным. Пустота объектива «быть красным 
или не быть красным» есть следствие того, что его выполняют все предметы, т. е. не происходит детерминации особого класса предметов. Это делает законы логики (тавтологии) неинформативными в предметно-теоретическом смысле, хотя они и остаются элементом мыслительных процессов [13, с. 12, 69-70].

Обратим внимание на то обстоятельство, что следствием из теорем 66 и 67.2 равенство $[T]=\varnothing$ становится уже независимо от гипотез, принимаемых при определении $G c(\alpha, \beta)$. Иными словами, $[T]=\varnothing$ есть теорема, вытекающая из определения $G c(\alpha, \beta)$. Получается, что введенное в определение родственности по следствиям ограничение, имеющее целью обеспечить релевантность следования, распространилось на всю систему и на ее центральное логическое отношение «обусловливать». Это нарушает адекватность построения Малли логике высказываний. Из частного случая аксиомы 2 вида $T \rightarrow T$ следует $[T] \subseteq[T]$, т. е. все законы логики следуют друг из друга, поэтому, хотя из их совокупности не следует ничего иного, кроме нее самой, утверждать пустоту следствий из $T$ невозможно. Чтобы стало верным равенство $[T]=\varnothing$, необходимо иначе определить понятие совокупности следствий объектива $\alpha$, а именно представить его, по меньшей мере, как совокупность следствий из $\alpha$, отличных от $T$, т. е., в каком-то смысле, нетривиальных следствий. Уточняя понимание нетривиальности, мы столкнемся с логически эквивалентными объективами, а также с теми, которые лишь обусловливаются $\alpha$. Значит, стремясь к нетривиальности, в новом определении совокупности следствий $\alpha$ следует оставить именно более слабые, неэквивалентные ему следствия. В этом случае действительно следствия $T$ окажутся пустым множеством.

Насколько оправданно ограничение, вводимое Малли? Для ответа на этот вопрос сопоставим классическому пониманию следования, для которого $[T]=T$, его «релевантизированный» вариант, который будет радикальнее варианта Малли, и сам вариант Малли, где $[T]=\varnothing$.

При классическом понимании следования определения Малли должны быть модифицированы, что приводит к следующему перечню определений и теорем: 
68. Если $[\alpha] \cap[\beta]=[T]$ и $\left.\left(P^{-[\alpha]}\right] \cap P^{-[\beta]}\right)=\varnothing$, то $\alpha$ и $\beta$ чужды по следствиям.

68.0. Если $\alpha$ и $\beta$ чужды по следствиям, то $[\alpha] \cup[\beta]=P$ и $\left.\left(P^{-[\alpha]}\right] \cup P^{-[\beta]}\right)=P$, иными словами, чуждые по следствиям объективы разбивают $P-[T]$ на два класса.

68.1. Если $\alpha$ и $\beta$ чужды по следствиям, то

$\left.G c(\alpha, \beta)=([\alpha] \cap[\beta]) \cup\left(P^{-[\alpha]}\right] \cap P^{-[\beta]}\right)=[T] \cap \varnothing=\varnothing$,

$U c(\alpha, \beta)=\left([\alpha] \cap P^{-[\beta]}\right) \cup\left([\beta] \cap P^{-[\alpha]}\right)=[F]=P$.

68.2. $G c(\alpha, \beta) \leftrightarrow \neg U c(\alpha, \beta)$.

68.3. $G c(T, F)=([T] \cap[F]) \cup\left(P^{-[T]} \cap P^{-[F]}\right)=[T] \cup N$.

68.4. $U c(T, F)=\left([T] \cap P^{-[T]}\right) \cup\left([F] \cap P^{-[F]}\right)=\varnothing$.

68.5. $\left.G c(\alpha, \neg \alpha)=([\alpha] \cap[\neg \alpha]) \cup\left(P^{-[\alpha]}\right] \cap P^{-[\neg \alpha]}\right)=[T] \cap \varnothing=\varnothing$.

68.6. $(\alpha \rightarrow \beta) \leftrightarrow G c(\alpha, \beta)=[\beta] \cup P^{-[\alpha]}$.

В этих теоремах используется классическая семантика следования, так что теорема 68.6 дает семантические условия материальной импликации.

68.7. $(\alpha \rightarrow \beta) \leftrightarrow U c(\alpha, \beta)=[\alpha] \cap P^{-[\beta]}-$ условие ложности импликации.

68.8. Независимость $\alpha$ и $\beta$ есть их различие по следствиям, за исключением следствий, эквивалентных $T$. В самом деле, если неверно, что $\alpha \rightarrow \beta$ и неверно, что $\beta \rightarrow \alpha$, то $[\beta] \not \subset[\alpha]$ и $[\alpha] \not \subset[\beta]$. Тогда $U c(\alpha, \beta)=\left([\alpha] \cap P^{-[\beta]}\right) \cup\left(P^{-[\alpha]} \cap[\beta]\right)=n \cup m$, такие, что $\alpha \in n, \beta \in m$. Верно и обратное.

Перейдем теперь к более «релевантной» версии следования. Обозначим как $* \alpha-$ класс эквивалентности $\alpha$, и пусть $N={ }_{D f}$ $P-(* T \cup * F)$. В целях сопоставления результатов двух указанных пониманий понятия совокупности следствий объектива $\alpha$ будем рассматривать как $[\alpha]$ классическую совокупность, в то время как совокупность нетривиальных следствий $\alpha$ обозначим как $\langle\alpha\rangle$ и определим следующим образом:

$\langle\alpha\rangle={ }_{D f}\{\beta: \alpha \rightarrow \beta \wedge \neg(\alpha \leftrightarrow \beta) \wedge(\neg T \rightarrow \beta)\}$.

Очевидно, что

67.3. $\langle\alpha\rangle \subseteq[\alpha]$,

67.4. $\langle T\rangle=\varnothing$.

Если учесть, что $F$ - произвольный всегда ложный объектив, $\langle F\rangle$ не может содержать ни одного такого объектива, т. е.

67.5. $\langle F\rangle=P-(* T \cup * F)=N$. 
Пусть для некоторого $\alpha$ существует $\beta \in\langle\alpha\rangle$, такое, что $\beta \leftrightarrow F$. Тогда верен объектив $\alpha \rightarrow F$, откуда $T \rightarrow \neg \alpha$, а значит, $\neg \alpha \in$ $\langle T\rangle$, что невозможно в силу 67.4. Значит, любой $\beta$ такой, что $\beta \in\langle\alpha\rangle$ не является ложным объективом. Тогда верно

67.6. $\langle\alpha\rangle \subseteq N-* \alpha$.

Введение $\langle\alpha\rangle$ в систему Малли сразу же приводит к тому, что перестает быть верной терема 66. Легко можно убедиться в том, что из гипотезы $G c(\alpha, \beta)=P-U c(\alpha, \beta)$ следует $\langle\alpha\rangle=\langle\beta\rangle$, что в общем случае неверно.

Исследуем теперь, какие результаты дадут нам указанные три подхода при применении к формулам, выражающим парадоксы материальной импликации.

Рассмотрим объектив $\alpha \rightarrow T$, где $\alpha$ нейтральный, т. е. не истинный, и не ложный объектив, понимая $[\alpha]$ как совокупность следствий в классическом смысле, а $\langle\alpha\rangle$ в соответствии с вышеприведенным определением. Итак: $\alpha \rightarrow T-$ гипотеза. $(\alpha \rightarrow T) \leftrightarrow[T] \subseteq[\alpha]$, теорема 61.1. $[T] \subseteq[\alpha]$, откуда $P^{-[\alpha]} \subseteq P^{-[T]}$, откуда $P^{-[\alpha]} \cap P^{-[T]}=P^{-[T]}$. Поскольку $\alpha \cap T=T$, получаем $G c(\alpha, T)=([\alpha] \cap[T]) \cup\left(P^{-[\alpha]} \cap P^{[-T]}\right)=[T] \cup P^{-[\alpha]}$. Иными словами, совокупность совместных следствий $\alpha$ и $T$ дает классические условия выполнения объектива $\alpha \rightarrow T$ : он выполняется на классе вещей, выполняющих совокупность объективов $[T] \cup P^{-[\alpha]}$. У Малли $G c(\alpha, T)=P^{-[\alpha]}$.

Сохранится ли объектив $\alpha \rightarrow T$, если аналогичное рассуждение провести для $\langle\alpha\rangle$ ? Примем гипотезу $\alpha \rightarrow T$. В силу определения $\langle\alpha\rangle, T \notin\langle\alpha\rangle$. Отсюда, $\neg(\alpha \rightarrow T)-$ противоречие с гипотезой, значит $\neg(\alpha \rightarrow T)$. Кроме того $G c(\alpha, T)=$ $(\langle\alpha\rangle \cap\langle T\rangle) \cup\left(P^{-\langle\alpha\rangle} \cap P^{-\langle T\rangle}\right)$. Это значит, что объектив $\alpha \rightarrow T$ не является всегда выполнимым, а оказывается эквивалентным $\neg \alpha$; один из парадоксов материальной импликации - «истина следует из чего угодно» - не верифицируется ни при $[\alpha]$, ни при $\langle\alpha\rangle$. Это совпадает с результатом, полученным по определениям Малли.

Проверим объектив $\alpha \rightarrow F$. К каким следствиям приведет гипотеза $\alpha \rightarrow F$ и может ли нейтральный $\alpha$ обусловливать $F$ ? Пусть $A=\langle\alpha\rangle$. Гипотеза: $\alpha \rightarrow F .(\alpha \rightarrow F) \leftrightarrow\langle F\rangle \subseteq\langle\alpha\rangle$ теорема 61.1 для нетривиальных следствий. $\langle F\rangle \subseteq\langle\alpha\rangle$, откуда $N \subseteq\langle\alpha\rangle$. 
Но $\alpha \in N$, и в силу определения $\langle\alpha\rangle \subseteq N$. Тогда $N=\langle\alpha\rangle$. Это следствие, очевидно, неприемлемо, как в связи с возникающими противоречиями (для произвольного нейтрального $\beta$ ), так и потому, что $* \alpha \subseteq N$, но $* \alpha \not \subset\langle\alpha\rangle$.

Построим $G c(\alpha, F)$. В силу определения $N: G c(\alpha, F)=(\langle\alpha\rangle \cap$ $\langle F\rangle) \cup\left(P^{-\langle\alpha\rangle} \cap P^{-\langle F\rangle}\right)=(A \cap N) \cup((P-A) \cap(P-N))=(A \cap$ $N) \cup(P-(A \cup N))$. В силу $67.6 A \subseteq N$, но в силу полученного выше $N=\langle\alpha\rangle$, и, поскольку $P^{-\langle F\rangle}=P-N=* \mathrm{~T} \cup * F$, получаем: $(A \cap N) \cup(P-N)=N \cup(P-N)=N \cup * \mathrm{~T} \cup * F=P$, т.е. $G c(\alpha, F)=$ $P$. Этот результат означает, что $\alpha \rightarrow F$ всегда истинно, откуда немедленно следует, что $\alpha=F$. Но это противоречит исходной гипотезе о нейтральности $\alpha$, а значит, $\alpha \rightarrow F$ при нейтральном $\alpha$ всегда невыполненно. Отсюда следует, что как при $[\alpha]$, так и при $\langle\alpha\rangle$ верно, что $(\alpha \rightarrow F) \leftrightarrow(\alpha=F)$.

Проверим теперь, как поведет себя при $\langle\alpha\rangle$ объектив $F \rightarrow \alpha-$ другой парадокс материальной импликации, гласящий «из лжи следует все, что угодно». Как и выше, сопоставим результаты для $[\alpha]$ и для $\langle\alpha\rangle$. Гипотеза: $F \rightarrow \alpha .(F \rightarrow \alpha) \leftrightarrow[\alpha] \subseteq[F]$, теорема 61.1. $[\alpha] \subseteq[F]$, откуда $P^{-[F]} \subseteq P^{-[\alpha]}$. Тогда, поскольку $[F]=$ Р и $P^{-[F]}=\varnothing, G \mathrm{c}(F, \alpha)=([F] \cap[\alpha]) \cup\left(P^{-[F]} \cap P^{-[\alpha]}\right)=[\alpha] \cup$ $\left(\varnothing \cap P^{-[\alpha]}\right)=[\alpha] \cup \varnothing=[\alpha]$. Получено условие выполнения $\alpha$. При этом: $U \mathrm{c}(F, \alpha)=\left([F] \cap P^{-[\alpha]}\right) \cup\left(P^{-[F]} \cap[\alpha]\right)=P^{-[\alpha]} \cup \varnothing=P^{-[\alpha]}$, что дает нам условия невыполнения $F \rightarrow \alpha$, совпадающие, как мы видим, с условиями невыполнения $\alpha$. У Малли $G c(F, \alpha)=$ $[\alpha]$.

Итак, уже для случая $[\alpha]$, и при том, что все используемые Малли определения сохраняются (в рассуждении не затрагивалась совокупность $[T]$ ), объектив $F \rightarrow \alpha$ не становится всегда истинным, но оказывается эквивалентным $\alpha$, что не соответствует семантике материальной импликации.

Рассмотрим теперь объектив $F \rightarrow \alpha$ для случая $\langle\alpha\rangle$. Гипотеза: $F \rightarrow \alpha .(F \rightarrow \alpha) \leftrightarrow\langle\alpha\rangle \subseteq\langle F\rangle$, теорема 61.1. для нетривиальных следствий. $\langle\alpha\rangle \subseteq\langle F\rangle$ и, поскольку $\langle F\rangle=N,\langle\alpha\rangle \subseteq N$. Тогда, $P^{-[F]} \subseteq P^{-[\alpha]}$, и, поскольку $P^{-[F]}=* T \cup * F$, и $* T \subseteq P^{-[\alpha]}$, получаем $G c(F, \alpha)=(\langle F\rangle \cap\langle\alpha\rangle) \cup\left(P^{-[F]} \cap P^{-[\alpha]}\right)=(N \cap\langle\alpha\rangle) \cup\left(P^{-[F]} \cap\right.$ $\left.P^{-[\alpha]}\right)=\left(N \cup P^{-[F]}\right) \cap\left(N \cup P^{-[\alpha]}\right) \cap\left(\langle\alpha\rangle \cup P^{-[F]}\right) \cap\left(\langle\alpha\rangle \cup P^{-[\alpha]}\right)=$ $(N \cup * T \cup * F) \cap\left(N \cup P^{-[\alpha]}\right) \cap(\langle\alpha\rangle \cup(* T \cup * F)) \cap\left(\langle\alpha\rangle \cup P^{-[\alpha]}\right)=$ 
$P \cap\left(N \cup P^{-[\alpha]}\right) \cap(\langle\alpha\rangle \cup * T \cup * F) \cap P=\left(N \cup P^{-[\alpha]}\right) \cap(\langle\alpha\rangle \cup$ $* T \cup * F)=* T \cup\{\beta: \beta \in * F \& \beta \in\langle\alpha\rangle\}$. Если существует такое $\beta$, то в силу сказанного выше $\alpha=F$, и тогда получаем $(N \cup * T \cup * F) \cap(N \cup * T \cup * F)=P$. Это значит, что объектив $F \rightarrow F$ всегда выполним, и это частный случай $\alpha \rightarrow \alpha$. Если же такого $\beta$ не существует, то $G c(F, \alpha)=* T$. При этом $U c(F, \alpha)=$ $\left(\langle F\rangle \cap P^{-[\alpha]}\right) \cup\left(\langle\alpha\rangle \cap P^{-[F]}\right)=\left(N \cap P^{-[\alpha]}\right) \cup(\langle\alpha\rangle \cap(* T \cup * F))=$ $(N \cup\langle\alpha\rangle) \cap(N \cup * T \cup * F) \cap\left(P^{-[\alpha]} \cup\langle\alpha\rangle\right) \cap\left(P^{-[\alpha]} \cup * T \cup * F\right)=$ $(N \cup\langle\alpha\rangle) \cap \mathrm{P} \cap \mathrm{P} \cap\left(P^{-[\alpha]} \cup * T \cup * F\right)=(N \cup\langle\alpha\rangle) \cap\left(P^{-[\alpha]} \cup * T \cup * F\right)$. Поскольку $\langle\alpha\rangle \subseteq\langle F\rangle$, получаем $N \cap\left(P^{-[\alpha]} \cup * T \cup * F\right)=N \cap P^{-[\alpha]}=$ $\{\beta: \beta \notin\langle\alpha\rangle \& \beta \in N\}$. Тем самым дано стандартное условие выполнения объектива $F \rightarrow \alpha$, указывающее на его выполнение в тех случаях, когда выполнено $T$, т. е. не имеет места $F$. Условия невыполнимости также адекватны классическим. Но при этом теорема 66 не действует.

Наконец, выясним статус объектива $F \rightarrow T$ при принятии классического и неклассического следования. Гипотеза: $F \rightarrow T$. $(F \rightarrow T) \leftrightarrow[T] \subseteq[F]$, теорема 61.1. $[T] \subseteq[F]$, откуда $\mathrm{P}^{-[F]} \subseteq$ $P^{-[T]}$. Тогда, поскольку $[F]=P, P^{-[F]}=\varnothing,[T]=[T]$, получаем $G \mathrm{c}(F, T)=([F] \cap[T]) \cup\left(P^{-[F]} \cap P^{-[T]}\right)=[T] \cup\left(\varnothing \cap P^{-[T]}\right)=[T] \cup \varnothing=$ $[T]$. Таким образом, при классическом определении следования условия выполнения $F \rightarrow$ Т совпадают с условиями выполнения $T$. У Малли $G c(F, T)=\varnothing$.

Обратимся теперь к неклассическому определению. Гипотеза: $F \rightarrow T .(F \rightarrow T) \leftrightarrow\langle T\rangle \subseteq\langle F\rangle$, теорема 61.1. $\langle T\rangle \subseteq\langle F\rangle$, откуда $P^{-\langle F\rangle} \subseteq P^{-\langle T\rangle}$. Тогда, поскольку $\langle F\rangle=N, P^{-\langle F\rangle}=* F \cup * T$, $\langle T\rangle=\varnothing$, получаем $G c(F, T)=(\langle F\rangle \cap\langle T\rangle) \cup\left(P^{-\langle F\rangle} \cap P^{-\langle T\rangle}\right)=$ $\varnothing \cup((* F \cup * T) \cap P)=\varnothing \cup * F \cup * T=* F \cup * \mathrm{~T}$. При этом $U c(F, T)=$ $\left(\langle F\rangle \cap P^{-\langle F\rangle}\right) \cup\left(\langle T\rangle \cap P^{-\langle T\rangle}\right)=(N \cap(* F \cup * T)) \cup \varnothing=\varnothing \cup \varnothing=\varnothing$. Это значит, что $F \rightarrow T$ выполняется вместе с всегда истинными, а также с всегда ложными объективами, что противоречит как классическому, так и неклассическому представлениям.

Дальнейшее исследование примеров для выявления следствий использования классического определения следования и определения нетривиального следования не принесет новых результатов. Выше мы установили следующее: классическое определение следования, при котором $[T]=* T \neq \varnothing$, не верифицирует объ- 
ективы $\alpha \rightarrow T, F \rightarrow \alpha$ и $F \rightarrow T$, отождествляя условия их истинности с истинностью или ложностью одной из их компонент, а именно указывая на условие невыполнения антецедента и (или) выполнения консеквента. В частности, $(\alpha \rightarrow T) \leftrightarrow \neg \alpha$, $(F \rightarrow \alpha) \leftrightarrow \alpha,(F \rightarrow T) \leftrightarrow T$. Используя определение нетривиального следования и исходя из $[T]=\varnothing$, также нельзя обосновать универсальную выполнимость $(\alpha \rightarrow T),(F \rightarrow \alpha)$ и $(F \rightarrow T)$. Представим все полученные данные в таблице:

Таблица 1. Сопоставление совокупностей совместных следствий объективов

\begin{tabular}{|l|l|l|l|}
\hline Объектив & $\begin{array}{l}\text { Gс по классиче- } \\
\text { скому определе- } \\
\text { нию }\end{array}$ & $\begin{array}{l}\text { Gс по опре- } \\
\text { делению } \\
\text { Малли }\end{array}$ & $\begin{array}{l}\text { пс по опреде- } \\
\text { лению нетри- } \\
\text { виальных } \\
\text { следствий }\end{array}$ \\
\hline$\alpha \rightarrow T$ & {$[T] \cup P^{-[\alpha]}$} & $P^{-[\alpha]}$ & $P^{-\langle\alpha\rangle}$ \\
\hline$F \rightarrow \alpha$ & {$[\alpha]$} & {$[\alpha]$} & $* T$ \\
\hline$F \rightarrow T$ & {$[T]$} & $\varnothing$ & $* F \cup * T$ \\
\hline
\end{tabular}

Мы видим, что определение нетривиальных следствий как более сильное, нежели определение Малли, приводит к контринтуитивным результатам в случае с объективом $F \rightarrow T$. Связано это с равенством $\langle F\rangle=N$, разрушающим всякую связь $\langle F\rangle$ и $* F$. Но это есть только результат применения определения $\langle\alpha\rangle \mathrm{K}$ $F$. Определения же Малли дают результаты близкие к классическому варианту. Лишь в случае с $F \rightarrow T$ различие существенно. Все это позволяет сделать вывод о том, что вводимое Малли равенство $[T]=\varnothing$ не дает существенных преимуществ при релевантизации отношения следования, но нарушает соответствие его системы классической логике.

Стремление Малли построить релевантную теорию следования, которая могла бы адекватно отражать разнообразные аспекты предметно-теоретического понимания отношения обусловливания, наталкивается на те же проблемы, с которыми 
столкнулся К. И. Льюис ${ }^{11}$, а вслед за ним и другие логики, работавшие со строгой импликацией и модальностями: исследование сложных контекстов языка без развитого аппарата логической семантики крайне затруднительно. Малли считал, что построение релевантной теории следования в логике объективов может быть осуществлено при сохранении соответствия предметнотеоретической логики теории классов как формальной онтологии. Как показали более поздние исследования по модальной и релевантной логикам, достичь этого невозможно. Поставленная Малли задача может быть решена при построении интенсиональной логики предмета, в которой законы, выражающие свойства вещей, и законы, выражающие свойства объективов, будут должным образом дифференцированы.

\section{3 Интенсиональная логика предмета, реализующая установки Малли}

В интенсиональной логике предмета (IGL) мы будем иметь дело с объективами как с интенсиональными сущностями. Выше уже говорилось, что Малли понимает объектив как нечто аналогичное пропозиции, но не как предложение. При этом в построении Малли отсутствуют атомарные объективы, взаимная независимость которых лежит в основе семантики пропозициональной логики. Связано это с тем, что Малли стремился сохранить возможность использования разных способов задания одного и того же объектива разными языковыми формулировками, т. е. предложениями. Этим объясняется внимание к эквивалентным объективам, которые, будучи взяты как пропозиции, оказываются неразличимыми. В перспективе построения семантики для объективов можно будет попытаться выделить элементарные и неэлементарные объективы по аналогии с элементарными и неэлементарными формулами пропозициональной логики, но многообразие атомарных объективов должно быть задано многообразием предложений, так что логическая независимость объективов не будет сохраняться. Критерий тождества объективов - интенсиональное тождество - следует искать не в их

\footnotetext{
${ }^{11}$ Следует напомнить, что первая статья К. И. Льюиса, посвященная вопросу о релевантной импликации, появилась в 1912 году.
} 
функциональной эквивалентности, а в их собственных специфических чертах.

Будем понимать неопределенные объективы как предикаты - свойства. Определенный объектив или случай будем понимать как приписывание свойства вещи, которое будет получать характер процедуры проверки истинности соответствующего предложения, так что отождествление двух случаев станет возможным лишь при отождествлении соответствующих процедур, что потребует тождества предложений. Отождествление неопределенных объективов будет производиться с точностью до совпадения соответствующих им определенных. Этот подход лежит в русле традиции интенсиональной логики, берущей начало с систем логики смысла и денотата Алонзо Чёрча ${ }^{12}$, которая легла в основу интенсиональной логики Ричарда Монтегю. Необходимость использования смысла в семантическом анализе становится неизбежной, как только замена тождественных рассматривается как допустимая для косвенного (интенсионального) контекста операция. Смысл привлекается как дополнительный аспект, использование которого позволяет более точно дифференцировать значения выражений. Замена тождественных в косвенном контексте считается тогда приемлемой только в случае «сильного» тождества, предполагающего как совпадение денотатов, так и совпадение смыслов выражений. Чёрч трактовал смысл выражения как то, что усвоено, когда выражение понято [5, с. 31-32], или как то, что «определяет денотат» [5, с. 19]. Применительно к предложениям можно также считать, что знание смысла предложения означает знание условий, при которых оно истинно, или что знание смысла предложения есть знание предполагаемой процедуры проверки его истинности. Процедурная трактовка смысла может иметь и более сильную интерпретацию, которую дают Яннис Московакис и Фритц Хамм: «значение терма есть алгоритм, вычисляющий его денотат» $[10, \text { с. } 5]^{13}$.

\footnotetext{
${ }^{12}$ См.: [5], [8], [9].

${ }^{13}$ См. также: [16]. Первая попытка сблизить понятие смысла с понятием алгоритма принадлежит, по-видимому, Павлу Тихому, автору варианта интенсиональной логики, который был назван им «транспарентной логикой» [17]. Процедурное понимание смысла разделяет Маркус Крахт [11].
} 
Для реализации этого принципа используются мощные инструменты логической грамматики, которые позволяют представить выражения языка в форме, с которой может быть соотнесена алгоритмическая процедура. Перед нами подобная задача не стоит, и для нас достаточно знать, что процедурное понимание смысла реализуемо, и что какими бы ни были соответствующие процедуры, имеется возможность описывать их свойства, т. е. исследовать смысл как динамическую единицу, как нечто становящееся ${ }^{14}$ и структурированное.

B IGL, следующей в русле теории Малли, должны быть формализованы следующие понятия: бытие вещью, бытие объективом (предметом), определенность объектива, неопределенность объектива, универсальность объектива, пустота объектива, возможность объектива, необходимость объектива, обусловливание одним объективом другого.

Алфавит IGL: (1) $i, j, i_{1}, j_{1}, \ldots$ - индивидные константы, (2) $P, Q, R, P_{1}, \ldots$ - одноместные элементарные предикатные константы, (3) ᄀ, $\wedge, \vee, \rightarrow,=, \Rightarrow, E X, E M T, \square, /, \wedge$ : отрицание, конъюнкция, дизъюнкция, импликация, равенство, обусловливание, существование, пустота, необходимость, «быть подпрограммой», интенсионал.

B IGL не используются переменные и нет квантификации, поскольку для теории Малли центральной задачей было различение вещей и предметов, при котором в число последних попадали бы понятия. Поскольку IGL строится в традиции построения интенсиональных логик, восходящей к Ричарду Монтегю, теория квантификация всегда может быть добавлена.

Определения терма и формулы.

ОПРЕДЕЛЕНиЕ 1. Термами являются индивидные константы, а также интенсионалы. Если $A-$ константа или формула, то ${ }^{\wedge} A \in P R$ - семантическая программа установления денотата $A$, т. е. интенсионал $A$. В частности, если $A-$ элементарный предикат, то $\wedge^{\wedge} A \in P R^{E}$, т. е. $\wedge^{\wedge} A-$ это элементарный интенсионал. Интенсионал индивидной константы $\wedge_{i}$ есть сама эта

\footnotetext{
${ }^{14}$ Временной аспект развертывания значения отмечается в работе Крахта $[11$, с. $415-416]$.
} 
константа $i$. Термы, обозначающие интенсионалы сложных выражений, образуются следующим образом ${ }^{15}$ : ^ $(\neg B) \approx n e g\left({ }^{\wedge} B\right)$ - инверсия, $\wedge^{\wedge}(B \wedge C) \approx{ }^{\wedge} B$ and ${ }^{\wedge} C$ - последовательное вычисление, $\wedge^{\wedge}(\mathrm{B} \vee C) \approx{ }^{\wedge} \mathrm{B}$ or ${ }^{\wedge} C-$ параллельное вычисление, ${ }^{\wedge}(\mathrm{B} \rightarrow C) \approx^{\wedge} \mathrm{B}$ ent ${ }^{\wedge} C-$ логическое следование.

ОПРедЕЛЕНИЕ 2. $\mathrm{P}(i)$ - элементарная формула. Если $A$ и $B-$ формулы, $\wedge^{\wedge} A, \wedge B$ - интенсионалы, $C$ и $D$ - термы, то $\neg A, A \wedge B$, $A \vee B, A \rightarrow B, \wedge A(i), C=D, \wedge A \Rightarrow^{\wedge} B, E X(i), E M T\left(^{\wedge} A\right), \square^{\wedge} A$, $\wedge A / \wedge B\left({ }^{\wedge} \mathrm{B}-\right.$ подпрограмма программы $\left.{ }^{\wedge} \mathrm{A} »\right)$ - формулы.

Множество семантических программ $P R$ строится на основе множества элементарных семантических программ $P R^{\mathrm{E}}$, т. е. таких, компоненты которых сами не являются программами. Выше ${ }^{\wedge} B$ и ${ }^{\wedge} C-$ это компоненты более сложных программ.

ОПредЕЛЕНИЕ 3. Если ${ }^{\wedge} \alpha_{1}, \ldots,{ }^{\wedge} \alpha_{n}$ - элементарные интенсионалы или их инверсии, то $\left[{ }^{\wedge} \alpha_{1}, \ldots,{ }^{\wedge} \alpha_{n}\right]$ есть дефинитивный комплекс, компонентами которого являются указанные программы. $\left[{ }^{\wedge} \alpha_{1}, \ldots,{ }^{\wedge} \alpha_{n}\right]={ }_{D f} \wedge \alpha_{1}$ and $\ldots$ and ${ }^{\wedge} \alpha_{n}$.

Значение выражений языка IGL определяется относительно окрестностной модельной структуры $K=\langle W, R\rangle$, где $W$ - множество возможных миров, $R$ - отношение достижимости вида $W \longrightarrow s(s(W))^{16}$. R рефлексивно, т. е., если $X \in R(w)$, то $w \in X$. Относительно $K$ определяются модели, так что некоторая модель $\mathrm{M}=\left\langle K, D, P R^{E}, I, 2\right\rangle$, где $D$ - непустое множество возможных индивидов, $P R^{E}-$ непустое множество элементарных семантических программ (смыслов предикатов), $I-$ функция интерпретации констант, $2=\{1,0\}-$ сигналы. Иными словами, значение выражений IGL зависит от набора параметров $W, R, D, P R^{E}, I$.

В модели $M$ функция интерпретации $I$ задает значения индивидных констант на множестве возможных индивидов $D$ и для каждого мира $w$ определяет $D_{w}$ как непустой набор индивидов, действительных относительно мира $w$. Кроме того, $I$ задает значение элементарным интенсионалам, сопоставляя им элементарные семантические программы.

\footnotetext{
${ }^{15}$ Знак $\approx$ выражает синтаксическое тождество.

${ }^{16} s-$ функция порождения степень-множества.
} 
Выражение ${ }^{\wedge} A$ обозначает конечную последовательность знаков, представляющую собой описание программы вычисления значения $A$. Каждое выражение, имеющее интенсионал, имеет единственный интенсионал, причем никакие два синтаксически различные выражения не имеют одинаковых интенсионалов. Это достигается путем использования геделевых номеров выражений - как для самого $A$, так и для $\wedge^{\wedge} A$. Интенсионалы отождествляются лишь при совпадении их номеров, т. е. при побуквенном тождестве соответствующих программ. Семантическая программа в качестве исходных данных берет выражение $\alpha$ и выражение, описывающее данные семантические условия, и вычисляет для них результат вида $\langle\beta, n\rangle$, где $\beta$ - есть денотат выражения $\alpha$ для данных условий, а $n$ - геделев номер выражения $\alpha$; при этом $n \approx{ }^{n} \alpha$. Тем самым обеспечивается единственность смысла $\alpha$ и его нетождественность смыслу любого другого, синтаксически отличного от $\alpha$ выражения. Уникальные номера присваиваются также дефинитивным комплексам интенсионалов, для чего используется как функция от номеров компонент комплекса ${ }^{17}$.

Множество номеров интенсионалов обозначим как $G$. Если интенсионал является элементарным, т. е. имеет вид $\wedge$, то его номер совпадает с номером $P$, т. е. $g n(\wedge P)=g n(P)$. Тем самым утверждается единственность смысла для каждого элементарного предиката. Элементарность интенсионала означает, что его работа как семантической программы не сводится к работе какой-либо другой программы, т. е. он не имеет подпрограмм. Если интенсионал не является элементарным, то вычисление его номера происходит по номерам входящих в него в качестве компонент элементарных интенсионалов. Обозначим совокупность номеров элементарных интенсионалов как $G^{\mathrm{e}} \in G$. По $n \in G$ можно однозначно восстановить выражение как $\wedge^{\wedge} A$, так и $A$, а также все элементарные интенсионалы $\wedge^{\wedge} B_{1}, \ldots, \wedge^{\wedge} B_{m}$, являющиеся компонентами $\wedge A$. Предикаты «быть элементарной се-

\footnotetext{
${ }^{17}$ Отличный от описываемого способ построения интенсионалов представлен в работе [1]. Он также обеспечивает сильные условия тождества интенсионалов, но опирается на синтаксическую структуру исходных выражений.
} 
мантической программой» и «быть подпрограммой» являются вычислимыми и могут быть выражены в IGL.

Выражения IGL имеют значения, определяемые следующими условиями (знак $\longrightarrow$ используется для обозначения отображения):

1. $|i|_{\mathrm{M}, w}=|i|_{\mathrm{M}} \in D$.

2. $\left.\left.\right|^{\wedge} P\right|_{\mathrm{M}, w}=\mathrm{p} \in P R^{E}: D \longrightarrow 2 \times G$.

Лемма 1. Для любого сложного интенсионала ${ }^{\wedge} A$ или дефинитивного комплекса существует функиионально равный ему элементарный интенсионал $\wedge \alpha$. $B$ этом случае для любого $B$ uмеет место $\left.\left.\right|^{\wedge} A\right|_{\mathrm{M}, w}(B)=\left.\left.\langle\beta, n\rangle u\right|^{\wedge} \alpha\right|_{\mathrm{M}, w}(B)=\langle\beta, m\rangle$, где $n \neq m$.

ДокАЗАТЕЛЬСТво использует п. 3-7 определения значения.

ОПРедЕЛЕниЕ 4. Интенсионал $\wedge^{\alpha} \alpha$ называется сложным тогда и только тогда, когда существуют интенсионалы $\wedge \delta_{1}, \ldots, \wedge \delta_{n}$, являющиеся его компонентами, вычисление значения которых для некоторых данных есть условие вычисление значения ${ }^{\wedge} \alpha$ для этих данных.

Лемма 2. Всякий сложный интенсионал $\wedge_{\alpha}$ представим как дефинитивный комплекс элементарных интенсионалов $\left[\wedge \beta_{1}, \ldots, \wedge \beta_{n}\right]$, калдый из которых является компонентом $\wedge^{\wedge} \alpha$, так что $\wedge^{\wedge} \alpha u\left[\wedge \beta_{1}, \ldots, \wedge \beta_{n}\right]$ функиионально эквивалентнь.

ДоКАЗАТЕЛЬСТво проводится с использованием п. 7 определения значения и с использованием определения номера ${ }^{\wedge} \alpha$.

ОПРеДЕЛЕНиЕ 5. Объем предиката: $|P|_{\mathrm{M}, w}={ }_{D f}\{e \in D$ : $\left.\left|{ }^{\wedge} P\right|_{\mathrm{M}, w}(e)=\langle 1, n\rangle\right\}$.

ОПРЕДЕЛЕНИЕ 6. Определение истинности.

1. $M, w \vDash \mathrm{P} i$ iff $|i|_{\mathrm{M}} \in|P|_{\mathrm{M}, w}$.

2. $M, w \vDash \alpha=\beta$ iff $|\alpha|_{\mathrm{M}, w}$ есть $|\beta|_{\mathrm{M}, w}$.

3. $M, w \vDash^{\wedge} \mathrm{P} i$ iff $|\wedge P|_{\mathrm{M}, w}\left(|i|_{\mathrm{M}}\right)=\langle 1, n\rangle$, где $n-$ номер ${ }^{\wedge} P i$.

4. $M, w \vDash \neg A$ iff $M, w \not \models A$.

5. $M, w \vDash \mathrm{A} \wedge \mathrm{B}$ iff $M, w \vDash \mathrm{A}$ и $M, w \vDash \mathrm{B}$.

6. $M, w \vDash$ E $i$ iff $|i|_{\mathrm{M}} \in D_{w}$. 
7. $M, w \vDash E M T^{\wedge} \mathrm{A}$ iff если $M, w \vDash \wedge \mathrm{P} i$, то $|i|_{\mathrm{M}} \notin D_{w}$.

8. $M, w \vDash n e g^{\wedge} \mathrm{A}(i)$ iff $M, w \vDash \neg \wedge \mathrm{A}(i)$.

9. $M, w \vDash\left({ }^{\wedge} \mathrm{A}\right.$ and $\left.{ }^{\wedge} \mathrm{B}\right)(i)$ iff $M, w \vDash \wedge$ A $(i) \wedge^{\wedge} \mathrm{B}(i)$.

10. $M, w \vDash\left(\wedge \mathrm{A}\right.$ or $\left.{ }^{\wedge} \mathrm{B}\right)(i)$ iff $\left\{v: M, v \vDash \wedge \mathrm{A}(i) \vee^{\wedge} \mathrm{B}(i)\right\} \in R(w)$.

11. $M, w \vDash\left({ }^{\wedge} \mathrm{A}\right.$ ent $\left.{ }^{\wedge} \mathrm{B}\right)(i)$ iff $\left\{v: M, v \vDash \wedge \mathrm{A}(i) \rightarrow{ }^{\wedge} \mathrm{B}(i)\right\} \in$ $R(w)$.

12. $M, w \vDash\left({ }^{\wedge} \mathrm{A} \Rightarrow^{\wedge} \mathrm{B}\right)$ iff $\left\{v:|A|_{\mathrm{M}, v} \neq \varnothing,|B|_{\mathrm{M}, v} \neq \varnothing \neq W\right.$ и для любого $i$ верно $\left.M, v \vDash^{\wedge} \mathrm{A}(i) \rightarrow{ }^{\wedge} \mathrm{B}(i)\right\} \in R(w)$.

13. $M, w \vDash \square^{\wedge} \mathrm{A}$ iff $\{v$ : для любого $i$ имеет место $M, v \vDash$ $\wedge \mathrm{A}(i)\} \in R(w)$.

14. $M, w \vDash \wedge \mathrm{A} /{ }^{\wedge} \mathrm{B}$ iff $g n\left({ }^{\wedge} \mathrm{B}\right)$ восстанавливается по $g n(\wedge \mathrm{A})$.

ОПРЕДЕЛЕНиЕ 7. Содержательное противоречие: $Z^{\wedge} \mathrm{A} \Leftrightarrow D f$ существует ${ }^{\wedge} \beta$, такой что ${ }^{\wedge} \mathrm{A} \Rightarrow{ }^{\wedge} \beta$ и ${ }^{\wedge} \mathrm{A} \Rightarrow n e g^{\wedge} \beta$.

ОПРеДЕЛЕНИЕ 8. Логическое противоречие $I N C^{\wedge} \mathrm{A}: Z^{\wedge} \mathrm{A} \Leftrightarrow D f$ существует $\wedge \beta$, такой что ${ }^{\wedge} \mathrm{A} \rightarrow{ }^{\wedge} \beta$ и $\wedge$ A $\rightarrow n e g^{\wedge} \beta$.

Ограничения в определении значения для $\Rightarrow$ принимаются для того, чтобы исключить истинность формул $F \Rightarrow \alpha$ и $\alpha \Rightarrow T$. Кроме того, само утверждение об обусловливании, хотя и основывается на задаваемых моделью объемных отношениях предикатов, релятивизовано к миру, отражая тем самым эпистемический характер отношения обусловливания.

Немедленно обнаруживается, что если $\left[{ }^{\wedge} \beta_{1}, \ldots,{ }^{\wedge} \beta_{n}\right]-$ дефинитивный комплекс $\wedge^{\wedge}$, то общезначима формула $\wedge \alpha \leftrightarrow$ $\left[\wedge \beta_{1}, \ldots,{ }^{\wedge} \beta_{n}\right]$. Но этого нельзя сказать о формуле $\wedge \alpha \Leftrightarrow$ $\left[\wedge \beta_{1}, \ldots,{ }^{\wedge} \beta_{n}\right]$. Этому препятствуют ограничения в условиях истинности для $\Rightarrow$. Иными словами, если комплекс «быть сферическим телом белого цвета» обусловливает свойство «быть белым», то комплекс «быть белым круглым квадратом» не обусловливает ни одного свойства, но имеет в качестве своего обычного логического следствия «быть круглым квадратом» и «быть белым».

Рассмотрим условия истинности суждения «быть квадратом означает быть четырехугольником». Запишем его как ${ }^{\wedge} \mathrm{A} \Rightarrow{ }^{\wedge} B$. При условиях $M, w$ эта формула истинна, когда к $R(w)$ принадлежит совокупность миров, для которых выраженные ${ }^{\wedge} \mathrm{A}$ и ${ }^{\wedge} B$ предикаты непусты, $B-$ не универ- 
сален, и при любом $i$ истинно ${ }^{\wedge} \mathrm{A}(i) \Rightarrow{ }^{\wedge} B(i)$. Интенсионалы $\wedge^{\wedge} \mathrm{A}$ и $\wedge^{\wedge} B$ могут быть представлены как дефинитивные комплексы $\left[\wedge \alpha_{1}, \ldots, \wedge \alpha_{n}\right]$ и $\left[{ }^{\wedge} \beta_{1}, \ldots, \wedge \beta_{m}\right]$, относительно которых верно, что $\left[\wedge \alpha_{1}, \ldots, \wedge \alpha_{n}\right] \Rightarrow\left[\wedge \beta_{1}, \ldots, \wedge \beta_{m}\right]$, и при этом $\wedge \beta_{1}, \ldots, \wedge \beta_{m} \subseteq \wedge \alpha_{1}, \ldots, \wedge \alpha_{n}$. Так мы получаем семантику отношения $\Rightarrow$, использующую понятие информации. Но для общего случая это не имеет места, поскольку, несмотря на наличие связи $\left[{ }^{\wedge} \alpha_{1}, \ldots,{ }^{\wedge} \alpha_{n}\right] \Rightarrow\left[\wedge \beta_{1}, \ldots,{ }^{\wedge} \beta_{m}\right]$, совпадений $\alpha$ и $\beta$ может не быть, поскольку функциональная эквивалентность не является достаточным условием для тождества интенсионалов. В этом случае возможно установление синонимии тех или иных объективов из двух комплексов. Но важно подчеркнуть, что при истинности ${ }^{\wedge} \mathrm{A} \Rightarrow{ }^{\wedge} B$ для дефинитивных комплексов ${ }^{\wedge} \mathrm{A}$ и ${ }^{\wedge} B$ всегда существует такое их представление вида $\left[\wedge \alpha_{1}, \ldots, \wedge \alpha_{n}\right] \Rightarrow\left[\wedge \beta_{1}, \ldots, \wedge \beta_{m}\right]$, где $\alpha_{i}$ и $\beta_{j}$ элементарны, что $\wedge \beta_{1}, \ldots, \wedge \beta_{m} \subseteq \wedge \alpha_{1}, \ldots, \wedge \alpha_{n}$.

Иллюстрацией первого случая служат отношения интенсионалов понятий «быть квадратом» и «быть прямоугольником», если сопоставить им дефинитивные комплексы [«быть замкнутой геометрической фигурой, образованной равными отрезками прямых, пересекающихся под прямыми углами»], и [«быть замкнутой геометрической фигурой, образованной отрезками прямых, пересекающихся под прямыми углами»], соответственно.

B IGL реализуется «сильное» различение интенсионалов. Следуя определениям значения и истинности, мы сможем отождествить семантические программы, которые, во-первых, функционально эквивалентны, а во-вторых, в ходе нумерации получают одинаковые номера, т. е. являются смыслами одних и тех же выражений языка и как программы совпадают побуквенно. При работе с дефинитивными комплексами можно игнорировать перестановки интенсионалов внутри комплекса.

Важное для Малли различение вещи и предмета достигается путем отождествления вещей с индивидами, т. е. элементами $D$, в то время как к категории вещей относятся как элементы $D$, так и элементы $P R$, т. е. интенсионалы. Сигналы 0 и 1 принадлежат при этом к отдельной категории. 
Интенсиональные сущности или объективы Малли представлены в IGL термами, т. е. являются компонентами формул. Это дает возможность как характеризовать их свойства и отношения, так и помещать их в контекст эпистемических установок. В представленной версии IGL нет эпистемических операторов, но они могут быть определены с помощью инструментов окрестностной семантики. Это же можно сказать и о ключевых для Малли понятиях вещь и предмет. B IGL нет индивидных концептов, к числу вещей принадлежат примитивы формальной онтологии, а к числу предметов - интенсионалы. Различение тех и других с помощью контекстуальных определений легко достижимо. Таким образом, IGL позволяет формализовать тот контекст рассуждений, для формализации которого Малли построил свою логику, а именно контекст рассуждений о существующих, возможных и невозможных предметах, о мыслимых и реальных фактах, об обусловленности одних свойств и фактов другими.

\section{4 Выводы}

Исследование предметно-теоретической теории Малли позволяет сделать следующие выводы:

(1) Перенося содержательные принципы теории предмета Мейнонга в логику, Малли опирался на известную к его времени интерпретацию логики высказываний с помощью логической алгебры. Эта интерпретация нашла формальное выражение в принципе реципрокности, который в современных терминах должен быть описан как принцип семантического вынуждения истинности формул языка логики объективов свойствами и отношениями соответствующих сущностей формальной онтологии. Исследуя другие свойства объективов, Малли эффективно использует реципрокность, что позволяет считать метод его работы формально-семантическим.

(2) Малли постулировал адекватность содержащей отношение «обусловливать» логики объективов классической логике высказываний, полагая, что с помощью ограничений отношению обусловливания можно будет придать смысл, соответствующий понятию строгой или релевантной импликации. Эти ограниче- 
ния, не достигая цели, приводят к расхождению между системой Малли и логикой высказываний. При этом анализ применимости универсальных логических законов к предметам разных типов позволил Малли сформулировать различие между объективом и вещью, т. е. между интенсиональной и неинтенсиональной сущностью.

(3) Построенная система интенсиональной логики предмета IGL реализует замыслы Малли. В ней объективы становятся интенсионалами, трактуемыми как семантические программы. Используется сильный критерий тождества интенсионалов, которое верифицируется лишь при идентичности соответствующих семантических программ. Сами программы задаются с помощью идентифицирующей нумерации как последовательности знаков. Важное для Малли отношение обусловливания интерпретируется в окрестностной семантике и отличается от материальной импликации. B IGL могут быть также эксплицированы эпистемические установки субъектов, наличие которых необходимо для представления теоретико-познавательных идей Мейнонга и Малли о схватывании, предположении и утверждении объективов.

\section{Литература}

[1] Микиртумов И. Б. Логика предмета Эрнста Малли: между логическим следованием и понятием причины // Логика, язык и формальные модели / Под ред. Е. Н. Лисанюк, И. Б. Микиртумова и Ю. Ю. Черноскутова. СПб.: Издательство СПбГУ. 2012. С 105-115.

[2] Микиртумов И. Б. Теория смысла и интенсиональная логика. СПб, Издательство СПбГУ, 2006.

[3] Суздалев E. H. «Алексиус Мейнонг: теория предмета (логикофилософский анализ)», диссертация, СПбГУ, 1991.

[4] Суздалев E. Н. Эрнст Малли: от теории предметов к логистике // материалы научной конференции «Современная логика: проблемы теории, истории и применения в науке» (16-17 июня 1994 г.). Ч. 2. СПб, 1994. С. 39-41.

[5] Чёрч A. Введение в математическую логику. Пер. с англ. В. А. Чернявского. М.: «Издательство иностранной литературы», 1960.

[6] Brentano F. Psychologie vom empirischen Standpunkte. Bd. 1. Leipzig, Verlag von Duncker \& Humblot, 1874 
[7] Chernoskutov Yu. Yu. Logic and object theory in 19th century: from Bolzano to Frege // Logical Investigations. Vol. 19. M.-Spb.: C.G.I., 2013. P. P. 10-22.

[8] Church A. A Formulation of the Logic of Sense and Denotation // Structure, Method and Meaning. Essays in honor of H. M. Sheffer. New York: The Liberal Arts Press, 1951. P. 3-24.

[9] Church A. The Need for abstract Entities in semantic Analysis // Contributions to the Analysis and Synthesis of Knowledge / Proceedings of the American Academy of Arts and Sciences, 1951. Vol. 80. P. 100-112.

[10] Hamm F., Moschovakis Y. Sense and denotation as algorithm and value. Advanced course. ESSLLI $2010 \mathrm{CPH}$. http://www.math.ucla. edu/ ynm/lectures/es10.pdf (последнее обращение 14.01.2013).

[11] Kracht M. Gnosis // Journal of Philosophical Logic. Vol. 40, 2011. P. 397-420.

[12] Lisanyuk E. N. Deontic 'coctail' according to E. Mally's receipt // Logical Investigations. Vol. 19. M.-Spb.: C.G.I., 2013. P. 100-121.

[13] Mally E. Gegenstandstheoretische Grundlagen der Logik und Logistik. (Ergänzungsheft zu Band 148 der Zeitschrift für Philosophie und philosophische Kritik. Leipzig: Bath. 1912.

[14] Meinong A. Über Annahme. 2. Aufl. Leipzig, 1910.

[15] Meinong A. Über Möglichkeit und Wahrscheinlichkeit. Beiträge zur Gegenstandstheorie und Erkenntnistheorie. Leipzig, Verlag von J. A. Barth, 1915.

[16] Moschovakis $Y$. Sense and denotation as algorithm and value // Proceedings of the ASL meeting 1990, Helsinki. Lecture Notes in Logic (№2. P. 210-249) / J. Oikkonen, J. Väänänen. Berlin, Heidelberg, 1994.

[17] Tichy' $P$. Intensions in terms of Turing Machines // Studia Logica. 1969. Vol. 24. P. 7-25.

\section{References (transliteration)}

[1] Mikirtumov I.B. Logika predmeta Ernsta Malli: mezhdu logicheskim sledovaniem i ponjatiem prichiny // Logika, jazyk i formal'nye modeli / Pod. red. E.N. Lisanjuk, I.B. Mikirtumova i Ju.Ju. Chernoskutova. Spb.: Izdatel'stvo SPbGU, 2012. S. 105-115.

[2] Mikirtumov I.B. Teorija smysla i intensional'naja logika. Spb.: Izdatel'stvo SpbGU, 2006.

[3] Suzdalev E.N. Aleksius Mejnong: teorija predmetov: Logiko-filosofskij analiz // Diss... . kand. filos. nauk. SPbGU, 1991. 
[4] Suzdalev E.N. Ernst Malli: ot teorii predmetov k logistike // Materialy nauchnoj konferencii «Sovremennaja logika: problemy teorii, istorii i primenenija v nauke» (16-17 ijunja 1994 ). Ch. 2. SPb., 1994. S. 3941.

[5] Cherch A. Vvedenie v matematicheskuju logiku. Per. s angl. V.A. Chernjavskogo. M.: Izdatel'stvo inostrannoj literatury, 1960.

[6] Brentano F. Psychologie vom empirischen Standpunkte. Bd. 1. Leipzig, Verlag von Duncker \& Humblot, 1874

[7] Chernoskutov Y. Y. Logic and object theory in 19th century: from Bolzano to Frege // Logical Investigations. Vol. 19. M.-Spb.: C.G.I., 2013. P. 10-22.

[8] Church A. A Formulation of the Logic of Sense and Denotation // Structure, Method and Meaning. Essays in honor of H. M. Sheffer. New York: The Liberal Arts Press, 1951. P. 3-24.

[9] Church A. The Need for abstract Entities in semantic Analysis // Contributions to the Analysis and Synthesis of Knowledge / Proceedings of the American Academy of Arts and Sciences. 1951, Vol. 80. P. $100-112$.

[10] Hamm F., Moschovakis Y. Sense and denotation as algorithm and value. Advanced course. ESSLLI $2010 \mathrm{CPH}$.

http://www.math.ucla.edu/ ynm/lectures/es10.pdf (последнее обращение 14.01.2013).

[11] Kracht M. Gnosis // Journal of Philosophical Logic. Vol. 40, 2011. P. 397-420.

[12] Lisanyuk E. N. Deontic 'coctail' according to E. Mally's receipt // Logical Investigations. Vol. 19. M.-Spb.: C.G.I., 2013. P. 100-121.

[13] Mally E. Gegenstandstheoretische Grundlagen der Logik und Logistik. (Ergänzungsheft zu Band 148 der Zeitschrift für Philosophie und philosophische Kritik. Leipzig: Bath. 1912.

[14] Meinong A. Über Annahme. 2. Aufl. Leipzig. 1910.

[15] Meinong A. Über Möglichkeit und Wahrscheinlichkeit. Beiträge zur Gegenstandstheorie und Erkenntnistheorie. Leipzig, Verlag von J. A. Barth. 1915.

[16] Moschovakis $Y$. Sense and denotation as algorithm and value // Proceedings of the ASL meeting 1990, Helsinki. Lecture Notes in Logic (№2. P. 210-249) / J. Oikkonen, J. Väänänen. Berlin, Heidelberg, 1994.

[17] Tichy' P. Intensions in terms of Turing Machines // Studia Logica. 1969. Vol. 24. P. $7-25$. 